\title{
AGB stars in the Magellanic Clouds
}

\section{The $\mathrm{C} / \mathrm{M}$ ratio}

\author{
M.-R. L. Cioni ${ }^{1}$ and H. J. Habing ${ }^{2}$ \\ 1 European Southern Observatory, Karl-Schwarzschild-Straße 2, 85748 Garching bei München, Germany \\ 2 Sterrewacht Leiden, Niels Bohrweg 2, 2333 RA Leiden, The Netherlands
}

Received 12 November 2002 / Accepted 6 February 2003

\begin{abstract}
Regions of different metallicity have been identified in the Magellanic Clouds by using the ratio between Asymptotic Giant Branch stars of spectral type C and M. In the Large Magellanic Cloud the ratio appears to decrease radially while in the Small Magellanic Cloud (SMC) there is no clear trend, reflecting either the large extension of the SMC along the line of sight or a more complex star formation history. The distribution of the $\mathrm{C} / \mathrm{M}$ ratio is clumpy and corresponds to a spread in $[\mathrm{Fe} / \mathrm{H}]$ of 0.75 dex in both Clouds. There is an indication of increasing $\mathrm{C} / \mathrm{M}$ ratio, thus decreasing metallicity, towards the Bridge region connecting the two Clouds.
\end{abstract}

Key words. galaxies: Magellanic Clouds - stars: AGB and post-AGB

\section{Introduction}

Asymptotic Giant Branch (AGB) stars trace the intermediate age population of galaxies (ages between 1 and several Gyr). In near-infrared (near-IR) light they are often the brightest isolated objects in a galaxy and can be studied at distances beyond $1 \mathrm{Mpc}$. There are two main kinds of AGB stars: oxygenrich stars (more $\mathrm{O}-$ atoms than $\mathrm{C}$-atoms, spectral type $\mathrm{M}$ or $\mathrm{K}$ ) and carbon-rich stars (more $\mathrm{C}$-atoms than $\mathrm{O}$-atoms, spectral type $\mathrm{N}$ or $\mathrm{C}$ ). When the number of $\mathrm{O}$-atoms equals that of C-atoms the AGB star is of type S. It is not straight forward to identify S stars either photometrically or spectroscopically, and this introduces as well intermediate classes of AGB stars (e.g. MS and SC). Single stars enter the AGB phase as oxygenrich but may be converted into carbon-rich stars after several short episodes in which matter that has been enriched in carbon nuclei by nuclear fusion is dredged-up to the stellar surface; these episodes are called thermal pulses; stars that have become C-stars in this way are called "intrinsic" C-stars; they may all have $\mathrm{N}$-type spectra.

Searches for carbon and M-type stars in Baade's Window (Glass et al. 1999), in the solar neighbourhood (Mikami 1978) and in the Magellanic Clouds (i.e. Blanco et al. 1980; Feast \& Whitelock 1994) have shown that the ratio of number of carbon stars to that of oxygen-rich AGB stars varies strongly from one environment to another and that it correlates with metallicity in the sense: lower metallicity, more carbon stars. For example, Cook et al. 1986 (and references therein) derived a C/M ratio in seven gas-rich galaxies and found that this correlates very well inversely with the abundance ratio $\log [\mathrm{O} / \mathrm{H}]$ : higher abundance lower C/M ratio - see their Fig. 6.

Send offprint requests to: M.-R. L. Cioni, e-mail: mcioni@eso.org
Iben \& Renzini (1983) explain the correlation between metallicity and the $\mathrm{C} / \mathrm{M}$ ratio by three factors: (1) for lower metallicity the O-rich stars will turn into C-type AGB stars more easily and stay so for a longer time on the AGB because less carbon atoms need to be dredgedup for the transformation; (2) at lower metallicity the evolutionary tracks for AGB stars move to higher temperatures and the number of M5+ ${ }^{1}$ stars drops considerably; the abundance of $\mathrm{TiO}$ molecules is lower and the atmosphere is more transparent; (3) in very-low metallicity environments a posthorizontal-branch star may become a white dwarf without becoming first an AGB star ("AGB-manqué stars").

In this article we use the DCMC, the DENIS (Epchtein et al. 1997) Catalogue of point sources detected in the $I J K_{\mathrm{s}}-$ photometric bands in the direction of the Magellanic Clouds (Cioni et al. 2000a). We eliminate foreground stars, we select red stars with a luminosity above the tip of the RGB (=TRGB) and we use photometric colours to distinguish between C-type AGB stars and AGB stars of class M0+. We then study the variation of the $\mathrm{C} / \mathrm{M} 0+$ ratio over the face of each Magellanic Cloud. We exploit two advantages of the DCMC catalogue over earlier studies: full coverage of both Clouds (320 square degrees in the LMC and 150 in the SMC) and the inclusion of $\mathrm{M}$ giants of all M-type spectra (M0+).

By considering only AGB stars above the TRGB we eliminate stars with a carbon-type spectrum, but NOT on the AGB: in a binary in which the secondary is a white dwarf, the primary may have a $\mathrm{C}$-rich spectrum because it received enough $\mathrm{C}$-enriched material from the secondary when, in the past, that

\footnotetext{
${ }^{1}$ When the sample of M-giants consists of stars with spectral type M0 and later we will designate this as "M0+"; similarly we will use $\mathrm{M} 3+, \mathrm{M} 5+$ etc.
} 
was an AGB star. Such stars are called "extrinsic C-stars"; a thorough discussion of their properties is given by Jorissen (2003). All R-type carbon stars, CH-stars and Ba-stars seem to be such extrinsic stars. Carbon stars with a luminosity above that of the TRGB must be AGB stars and thus very probably intrinsic carbon stars.

In Sect. 2 we discuss the sample and our selection criteria and we present the $\mathrm{C} / \mathrm{M}$ ratio and compare it with the values obtained during previous researches. Section 3 addresses the metallicity distribution derived in other papers and we compare the Magellanic Clouds (MC) with other galaxies. Conclusions are given in Sect. 4.

\section{The distribution of M-type and C-type AGB stars in LMC and SMC}

\subsection{Selection of AGB stars from the DENIS catalogue}

We selected AGB stars from the DCMC survey following the same criteria as used in an earlier paper (Cioni et al. 2000b). Briefly: in each Cloud we select all stars that (1) have been detected in all three DENIS wavelength bands $\left(I J K_{\mathrm{S}}\right)$; that $(2)$ have an $I$-band magnitude above that of the TRGB $(I=14.54$ in the LMC and $I=14.95$ in the SMC) and (3) that have a red colour $(I-J)>(19.78-I) / 4.64$ separating MC stars from foreground objects. The same sample has been used in an earlier paper to discuss the morphology of each Cloud (Cioni et al. 2000b; see region B in their Fig. 1). The two samples contain almost all AGB stars above the TRGB; we only miss stars with a circumstellar shell thicker than $\left(J-K_{\mathrm{s}}\right)>2.4$. The evolution of stars with a thick circumstellar envelope is so fast that there are always very few of them. Experience with ISO programmes confirms that the number of stars with such thick shells is too small to affect our conclusions (Loup 1999). The LMC sample contains 32801 AGB stars; the SMC sample contains 7652 stars.

\subsection{Contamination by foreground stars}

Our selection criterium rejects most of the foreground stars (Cioni et al. 2000b). Does the remaining fraction of foreground stars affects our conclusion? In this paper Fig. 1 contains a faint extension to higher $(I-J)$ values at $\left(J-K_{\mathrm{s}}\right)=0.9$; from DENIS colour-colour diagrams of stars outside of the Magellanic Clouds it follows that these are as well foreground objects. Nevertheless, this plume of objects contains a small number of genuine Magellanic Cloud stars: faint super-giants of spectral type K or M (Nikolaev \& Weinberg 2000). Foreground stars, if uniformly distributed, will affect the ratio, but will not introduce variations in the ratio. Blanco \& McCarthy (1983) observed several fields covering a total of about 6 and 7 square degrees in the LMC and SMC, respectively (see Sect. 3.2), and sampled foreground areas at the same latitude. They found on average $10 \mathrm{M} 2$ foreground stars per field $\left(0.12 \mathrm{deg}^{2}\right)$. If the distribution of foreground stars is the same as shows the gray scale in Fig. 2 in Cioni et al. (2000b), the enhancement towards the East is also the distribution of early M-type foreground stars, we would expect to find a smaller ratio in this direction than

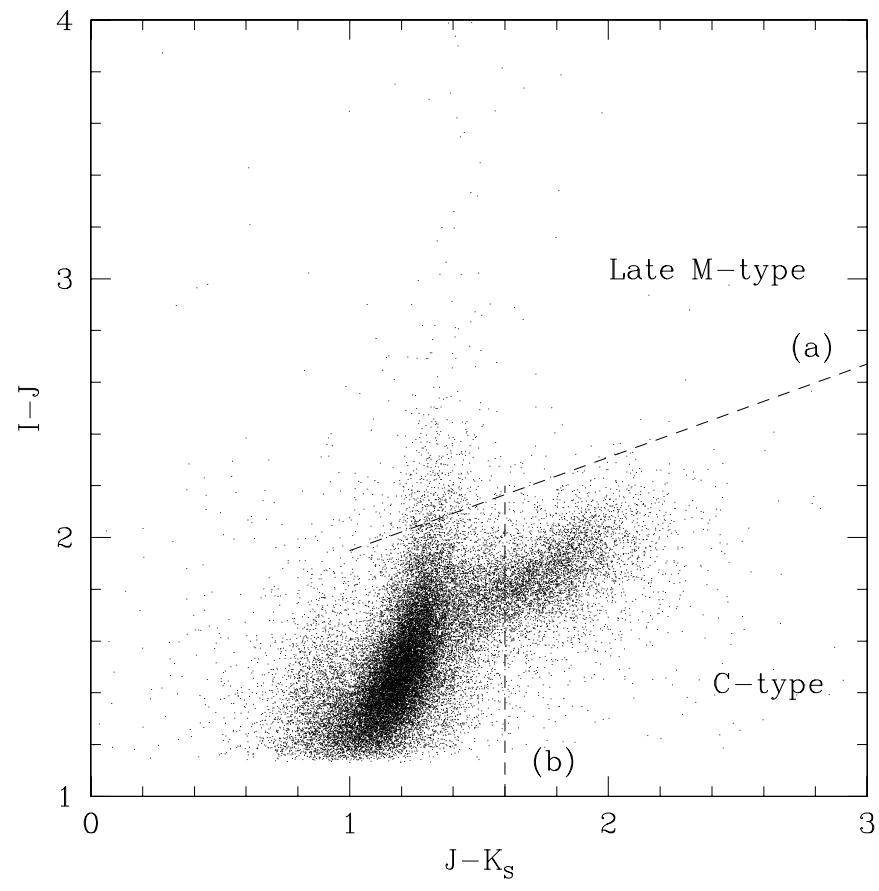

Fig. 1. DENIS colour-colour diagram of AGB stars in the LMC. Dashed line indicate the regions where upper M-type AGB and Ctype AGB stars can be discriminated according to Loup et al. (2002).

towards that directly opposite. But looking at Fig. 3 we do not find a noteworthy difference between the two regions. Thus we conclude that the distribution of foreground stars does not bias the $\mathrm{C} / \mathrm{M}$ ratio that we derive. The $\mathrm{C} / \mathrm{M}$ ratios, on the other hand, are lower limits, because there may be a foreground contamination by $\mathrm{M}$ dwarfs (Cook et al. 1986).

\subsection{Separating the M-type stars from the C-type stars}

Figure 1 shows all stars of the sample in a colour-colour diagram. A comparison one-by-one of a subsample of DCMC stars coinciding with stars with a spectral type derived from spectroscopy (Loup et al., in preparation) shows that the stars above the slanted dashed line (a) are all M-type giants of spectral type $\mathrm{M} 7+$ and the stars below this dashed line and to the right of the vertical line $\left(J-K_{\mathrm{s}}\right)=1.6$ (b) are all C-stars. This leaves the stars in the lower left part of the diagram below line (a) and left of line (b). We assume that we can separate the stars in M-type stars and in C-type stars in the LMC by the criterium $\left(J-K_{\mathrm{s}}\right)<$ or $>1.4$ (see Fig. 2) and in the SMC $\left(J-K_{\mathrm{s}}\right)=1.3$ will be the dividing line. We also assume that that the $\left(J-K_{\mathrm{s}}\right)$-colour is practically independent of spectral type whereas the $(I-J)$-colour is correlated strongly with the spectral type of the M-stars. This, for example has been found by Fluks et al. (1994): for M0-giants $(I-J)=1.14$ and $\left(J-K_{\mathrm{S}}\right)=1.03$ and for M9-stars these values are 4.06 and 1.25. Fluks' colours are based on stars in the solar neighbourhood. Therefore we calibrated our relation between spectral type and the colour $(I-J)$ on the results obtained by Blanco et al., Blanco \& McCarthy $(1980,1983)$ in the LMC and we correct the value of $(I-J)_{0}$ to 1.02 for M0-giants and to 1.99 for M7-giants. 
Table 1. C/M ratio in the Magellanic Clouds.

\begin{tabular}{lccccc}
\hline \hline Cloud & C & C/M0+ & C/M3+ & C/M5+ & C/M7+ \\
LMC & 7572 & 0.30 & 0.47 & 1.90 & 25.1 \\
SMC & 1643 & 0.27 & 0.87 & 9.28 & 48.3 \\
\hline
\end{tabular}

The location of the $\mathrm{C}$-star branch has been explained recently by accounting properly the molecular opacity in the stellar atmosphere when a star develops from an M-type star into a C-star (Marigo, Girardi \& Chiosi, accepted in A\&A). We conclude that a vertical line at $\left(J-K_{\mathrm{S}}\right)=1.4$ (the dashed line in Fig. 2) separates the M-type stars from the C-stars. The next conclusion is that in the area of the M-giants (left of the line $\left.\left(J-K_{\mathrm{s}}\right)=1.4\right)$ the $(I-J)$ value determines the M-type subclass: at $(I-J)=1.2$ the stars will be of spectral type M0; at $(I-J)=3.8$ they will be M9+. Close to the point $\left(I-J, J-K_{\mathrm{s}}\right)=(1.4,1.3)$ some C-stars will be mixed with the M-stars, but their fraction seems to be quite low. S-type AGB stars have near-IR colours similar to O-rich AGB stars and they cannot be disentangled in our diagrams; but there are always few around.

We apply this result in Fig. 2 , the $\left(K_{\mathrm{S}}, J-K_{\mathrm{S}}\right)$ colourmagnitude diagram of our LMC sample of AGB stars and obtain a sample of 25229 M-type stars and of 7572 C-stars. As remarked above, the first sample contains a small fraction of C-stars. For the SMC we obtain 6009 M-type and 1643 C-type AGB stars. The photometric accuracy of DENIS at the AGB magnitudes (i.e. for $M_{\mathrm{bol}}<-4$ ) is below 0.05 mag and the number of sources that have been put in the wrong section of Fig. 2 by photometric errors is negligible.

Using the $(I-J)$ colours of $\mathrm{M}$ giants as indicated by Blanco et al. (1980) and correcting for extinction $\left(A_{I-J}=0.16\right.$ for $E(B-V)=0.15$ as in Cioni et al. 2000c) we obtain the numbers of $\mathrm{C}-$-stars and the numbers of $\mathrm{M} 0+, \mathrm{M} 3+, \mathrm{M} 5+$ and $\mathrm{M} 7+$ stars. The numbers of giants of $\mathrm{C}$ and $\mathrm{M}$ spectral subtype with the corresponding ratio are given in, or can be easily obtained from, Table 1 . The table shows the population of early M-type AGB stars. The C/M0+ numbers are very similar for both LMC and SMC, which may indicate a similar average metallicity for both Clouds.

\subsection{Total number of carbon stars}

Blanco \& McCarthy (1983) expect 11000 carbon stars in the LMC and 2900 in the SMC. Groenewegen (2002) calculated from the Kontizas et al. (2001) survey of the LMC, that the carbon stars amount to 7750. Rebeirot et al. (1983) on the other hand found 1707 carbon stars in the SMC. These numbers are not very different from our estimated number of carbon stars based on the colour-magnitude selection criteria. We found, in the LMC, only $7572 \mathrm{C}$-type stars, and we think that we are complete, except for about $10 \%$ misclassifications (Cioni et al. 2001b) among the M0 and M1 giants. This would increase the number of possible C-type stars detection to 10094 and 2243 in the LMC and SMC, respectively, and the present C/M ratio would be thus underestimated. Other missing C-type stars are those with luminosities below the TRGB that might have

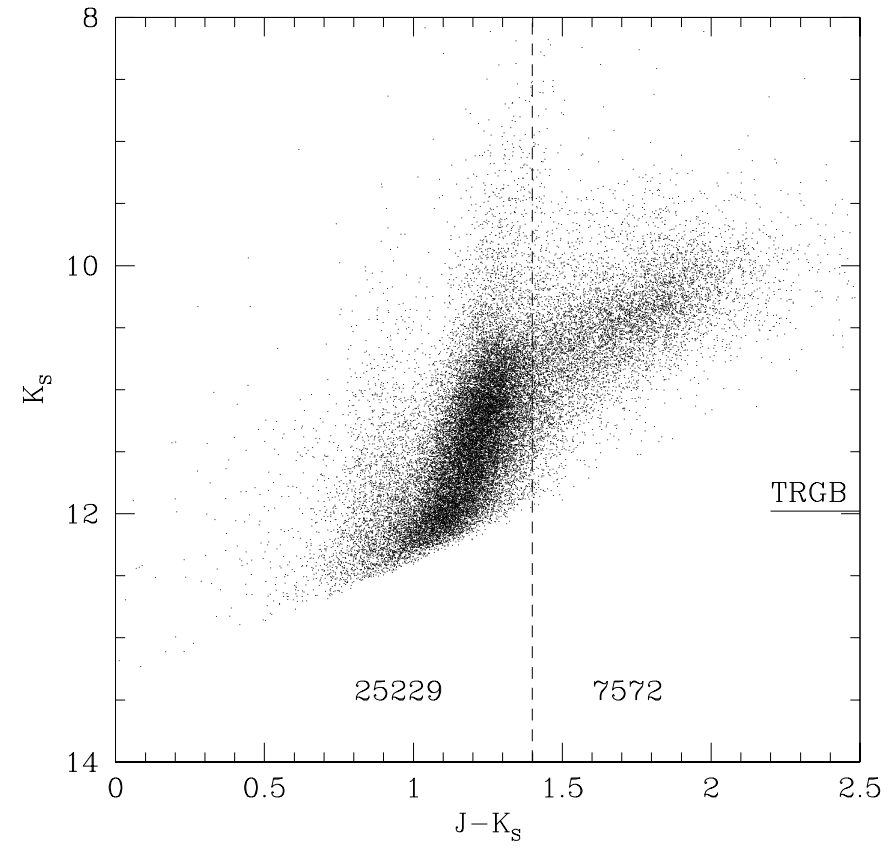

Fig. 2. Colour-magnitude diagram of stars in the LMC selected to be AGB stars above the TRGB $(I=14.54$, Cioni et al. 2000c $)$ and $I>$ $-4.64 \times(I-J)+19.78$ (Cioni et al. 2000b). The 7572 stars to the right of the dashed vertical line are carbon stars, whereas the 25229 stars to the left are M-type AGB stars.

weaker $\mathrm{CN}$ bands and may therefore have been missed as well on the red grism plates used by Blanco \& McCarthy (1983).

\subsection{The distribution of the $\mathrm{C} / \mathrm{M}$ ratio over the face of LMC and SMC}

From our samples of M-type and C-type AGB stars we have calculated $\mathrm{C} / \mathrm{M} 0+$ in all cells belonging to a grid a $100 \times 100$ of 0.04 square degrees over the face of the LMC and the SMC. Figures 3 and 4 show the ratios after boxcar average smoothing of width 2. The most outstanding feature is the ring of high $\mathrm{C} / \mathrm{M} 0+$ values around the outer edge of the LMC. In the SMC the distribution is quite clumpy. Both features are in contrast to what is found for the distribution of all (M+C-type) AGB stars (Cioni et al. 2000b): these all-AGB star distributions are smooth and regular and they have the highest densities in the center of each galaxy.

Are the structures in Figs. 3 and 4 real? We use $10^{4}$ squares and a sample of $25000 \mathrm{M}$-giants and $7000 \mathrm{C}$-stars, thus on average each bin contains $2 \mathrm{M} 0+-$ type stars and $0.7 \mathrm{C}$ star, and the ratio $\mathrm{C} / \mathrm{M} 0+$ is affected by noise due to small-number statistics. In the bin corresponding to the highest ratio there are $3 \mathrm{C}-$ and $1 \mathrm{M} 0+-$ star. In bins where $\mathrm{M}$-type stars were not detected, the $\mathrm{C}$-type number of stars was taken as an upper limit to the ratio, i.e. a ratio $\mathrm{C} / \mathrm{M} 0+=1$ was assumed. To check that the resulting pattern does not depend on the bin size we also made maps with $50 \times 50$ bins and obtained the same result. Now the bin with the lowest $\mathrm{C} / \mathrm{M}$ ratio contains $2 \mathrm{C}-$ and $4 \mathrm{M} 0+-$ type stars whereas the bin with the highest ratio 


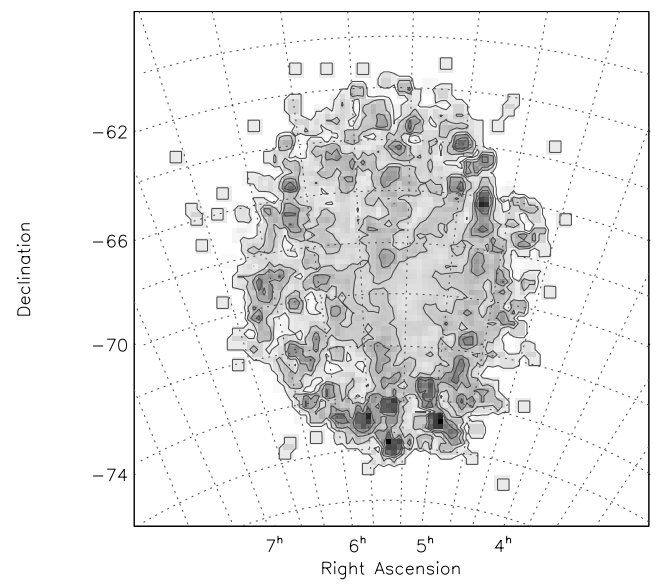

Fig. 3. The $\mathrm{C} / \mathrm{M} 0+$ ratio in the LMC. Contours are at: $0.1,0.25,0.4$, 0.55. Darker regions correspond to a higher ratio. Coordinates correpond to the $\mathrm{J} 2000$ system.

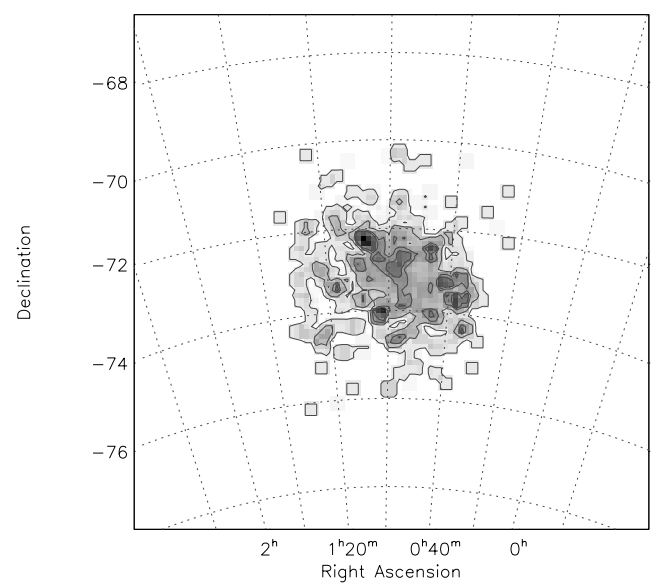

Fig. 4. The $\mathrm{C} / \mathrm{M} 0+$ ratio in the SMC. Contours are at: $0.1,0.3,0.5$, 0.6. Darker regions correspond to a higher ratio.

contains $7 \mathrm{C}$ - and $7 \mathrm{M} 0+-$ stars. We conclude that the variations seen in Figs. 3 and 4 are real.

In the LMC, apart from the ring, there is a small region of a relatively high $\mathrm{C} / \mathrm{M} 0+$ value at about $\alpha=5^{\mathrm{h}} 30^{\mathrm{m}}$ and $\delta=-67^{\circ}$. This structure points approximately to the middle of the bar and defines two lobes (NW \& SE) of lower $\mathrm{C} / \mathrm{M} 0+$ values and two other lobes (NE \& SW) of higher $\mathrm{C} / \mathrm{M} 0+$. The features may be related to the effect of the bar on the star formation history or vice versa as we do not know precisely when the bar has formed. Considering that the metal-poor component is probably old, the bar appears to be a fairly recent structure. The LMC bar extends approximately from $\alpha=5^{\mathrm{h}} 40^{\mathrm{m}}, \delta=-70^{\circ}$ to $\alpha=5^{\mathrm{h}} 00^{\mathrm{m}}, \delta=-69^{\circ}$.

In the SMC the situation is more complex - see Fig. 4. The fluctuations in this figure are real: the highest $\mathrm{C} / \mathrm{M}$ ratio is reached in a bin with $4 \mathrm{C}$-stars and $1 \mathrm{M} 0+-$ star and the lowest ratio in a bin with $16 \mathrm{C}-$ and $16 \mathrm{M} 0+-$ stars. The blob-like regions of high $\mathrm{C} / \mathrm{M} 0+$ do not form a regular pattern; there is a hint that $\mathrm{C} / \mathrm{M} 0+$ is higher in the external part of the central region. The lowest ratio is obtained in the so called "wing".
Probably the extension of the SMC along the line of sight contributes to the irregular distribution.

Around the centre of the SMC the $\mathrm{C} / \mathrm{M} 0+$ ratio is higher than inside the ring of the LMC. This is in agreement with earlier studies (e.g. Blanco et al. 1980) that show the metallicity of the AGB population in the SMC to be lower than in the LMC.

\subsection{Related previous studies}

In Fig. 2 most of the stars populating the (almost) vertical sequence to the left of the line $\left(J-K_{\mathrm{s}}\right)=1.4$ are of spectral type M0+. Blanco \& McCarthy (1983) discussed the ratios $\mathrm{C} / \mathrm{M} 2+, \mathrm{C} / \mathrm{M} 5+$ and $\mathrm{C} / \mathrm{M} 6+$ and found $0.2(0.63), 0.8(4.3)$, 2.2(13.8) for the entire LMC (SMC): the increase illustrates the existence of a large number of M-giants of early spectral type. In the earlier papers (e.g. Blanco et al. 1980) the samples are incomplete in these earlier types except in a few small fields mentioned below: M-giants of earlier spectral type have weak $\mathrm{TiO}$ and $\mathrm{VO}$ molecular bands and require spectra of very good quality to derive the subtype. Blanco \& McCarthy (1983) stated that the estimated number of M2-M4 stars may be in error by $20 \%$. The authors showed that an approximate linear relation exists between the frequencies of carbon stars and those of M2-M4 giants: for each carbon star there are 4.6 and $1.58 \mathrm{M} 2-\mathrm{M} 4$ stars in the LMC and in the SMC, respectively. Westerlund (1997) suggests that the most massive AGB stars could be of early type. These stars should exist in the Blanco fields as the immediate precursors of the detected Cepheid population.

Frogel \& Blanco (1990) detected stars with spectral types from M0 to M5 but only in about 0.03 square degrees in the Bar West field of the LMC. In the northern LMC Reid \& Mould (1985) spectroscopically identified O-rich stars of M0+ but only in six small fields (total area of 0.8 square degrees) in the northern LMC. They derive $\mathrm{C} /$ non- $\mathrm{C}$ ratios between 0.19 to 0.56 . The similarity with the range of values obtained from Fig. 3 proves the validity of our photometric selection criteria for $\mathrm{M} 0+$ stars and $\mathrm{C}$ stars.

Loup et al. (2002) conclude that in the region roughly indicated by $\left(J-K_{\mathrm{s}}\right) \approx 1.1$ and $(I-J)<2.0$ early M-type AGB stars are mixed with C-rich AGB (Fig. 1). If we apply the selection criteria given by the straight lines in Fig. 1 we obtain 4456 C-type and 765 M7+-type AGBs in the LMC. The distribution of the $\mathrm{C} / \mathrm{M} 7+$ ratio is strongly dominated by the distribution of C-type stars and the value of the ratio is high in the bar and progressively decreases to the outer parts. Using a coarser grid $(50 \times 50$ instead of $100 \times 100)$ as discussed in Sect. 2.5) we find a higher $\mathrm{C} / \mathrm{M} 7+$ ratio outside of the bar. In both cases the numbers of stars are low and the ratio can be calculated with confidence only in the inner part of the disk that is inside the metal poor ring identified in Fig. 3, thus closer to the bar. Above we have stated that AGB stars of the same luminosity but of lower metallicity have earlier spectra and this is further supporting our conclusion that the stars in the ring in the LMC have lower metallicity. 
Table 2. $\mathrm{C} / \mathrm{M}$ ratio in the $\mathrm{LMC}$ per ring.

\begin{tabular}{llcccccc}
\hline \hline $\mathrm{N}$ & Ring & $\mathrm{C}$ & $\mathrm{C}_{\text {red }}$ & $\mathrm{M} 0+$ & $\begin{array}{c}\mathrm{M} 6+ \\
\times \frac{A_{\text {RING }}}{A_{\text {TOT }}}\end{array}$ & $\begin{array}{c}\mathrm{C} / \mathrm{M} 0+ \\
\times \frac{A_{\text {RING }}}{A_{\mathrm{TOT}}}\end{array}$ & $\mathrm{C} / \mathrm{M} 6+$ \\
$(0)$ & $\rho<2.5^{\circ}$ & 3906 & 1668 & 12071 & 2236 & 0.045 & 0.104 \\
$(1)$ & $2.5^{\circ}<\rho<3.4^{\circ}$ & 1220 & 572 & 3836 & 734 & 0.038 & 0.092 \\
$(2)$ & $3.4^{\circ}<\rho<4.4^{\circ}$ & 1093 & 490 & 3288 & 586 & 0.058 & 0.145 \\
$(3)$ & $4.4^{\circ}<\rho<5.5^{\circ}$ & 832 & 398 & 2824 & 538 & 0.071 & 0.179 \\
$(4)$ & $5.5^{\circ}<\rho<6.7^{\circ}$ & 417 & 192 & 1881 & 271 & 0.072 & 0.231 \\
\hline
\end{tabular}

\section{A metallicity gradient in the LMC?}

The variation of the $\mathrm{C} / \mathrm{M} 0+-$ ratio may be caused by two effects: a variation in metallicity or a variation in the star formation rate depending on stellar mass. This second possibility will be discussed in a later paper; here we assume that the metallicity variation is the main cause of the $\mathrm{C} / \mathrm{M} 0+$ variation.

To investigate in more detail the variation of the (C/M0+)-ratio over the face of the LMC we calculated this ratio within segments of concentric rings as follows (the same procedure was adopted in van der Marel \& Cioni 2001): the centre is at $\left(\alpha=5^{\mathrm{h}} 29^{\mathrm{m}}, \delta=-69.5^{\circ}\right)$; the inner circle (i) has a radius of $2.5^{\circ}$, and the rings are (ii) between $2.5^{\circ}$ and $3.4^{\circ}$, (iii) between $3.4^{\circ}$ and $4.4^{\circ}$, (iv) between $4.4^{\circ}$ and $5.5^{\circ}$ and (v) between $5.5^{\circ}$ and $6.7^{\circ}$. Table 2 lists: (i) the number of the ring, (ii) inner and outer limit of the ring, (iii) the number of C-type and M-type stars as obtained from the global selection (C \& $\mathrm{M} 0+$ ); (iv) similar numbers from a sample using the more restrictive selection criteria derived by Loup et al. (e.g. Sect. 2.3), (v) the average of $\mathrm{C} / \mathrm{M} 0+$ and $\mathrm{C} / \mathrm{M} 6+$ multiplied by the fractional area covered by each ring $\left(A_{\mathrm{RING}} / A_{\mathrm{TOT}}\right)$. The average value of the surface density increases for larger distance from the center; excluding the very central region, the trend deduced from Fig. 3 is conserved.

If the outermost ring marks lower metallicity it must also contain relatively more early $\mathrm{M}$-giants than the central area. This is indeed the case: see Fig. 5, that shows the normalised number of O-rich sources as a function of $(I-J)$ colour in the central $\left(\rho<2.5^{\circ}\right)$ and in the outermost ring $\left(5.5^{\circ}<\rho<6.7^{\circ}\right)$ of the LMC. The panel on the upper right corner shows the same histograms with $\mathrm{C}$-type stars added; the latter produce a bump in the distribution at about $(I-J)=1.7$. In the outermost ring the distribution of the colours peaks at about $(I-J)=1.3$ and the histogram of the colours of the central area peaks at 1.5. At about $(I-J)=1.7$ (M5) there are almost twice as many sources in the center as in the outer ring. This is the effect that we predicted. Unfortunately we cannot exclude the possibility that the shift of the peak of the histograms is due to exinction. If we assume that the reddening decreases linearly with increasing radius with a step of $(I-J)=0.025$ in the range $0.1-0$ from the center to the outer regions, and we recalculate the $\mathrm{C} / \mathrm{M}$ ratio we obtain $0.33,0.59,2.94$ and 38.4, for the $\mathrm{C} / \mathrm{M} 0+, \mathrm{C} / \mathrm{M} 3+$, $\mathrm{C} / \mathrm{M} 5+$ and $\mathrm{C} / \mathrm{M} 7+$, respectively. In addition there are sources in the innermost bin at $(I-J)_{0}<1.02$ which would imply a spectral type earlier than M0. Thus we conclude that if a differential reddening between the center and the outskirts of the

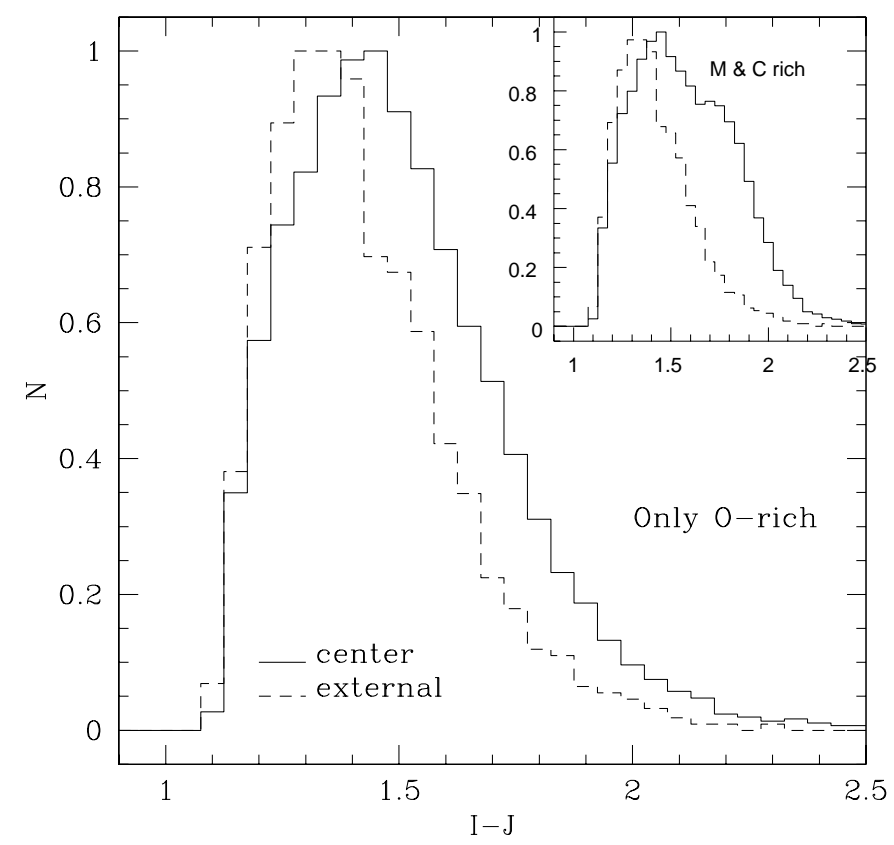

Fig. 5. Histograms of the distribution of the $(I-J)$ colour of sources in the central part (continuous line) and in the most external ring (dashed line) of the LMC. Regions are defined in the text. The central diagram includes only O-rich sources and the diagram on the upper right corner includes as well C-rich sources.

LMC is taken into account, giants in the center would have, on average, an earlier spectral type than giants in the outskirts.

The next step is to divide each ring in the LMC into eight segments of $45^{\circ}$ and labelled counterclockwise from 1 through 8 or by wind directions (E, SE, S, SW, W, NW, N and NE) per increasing angle. The first sector starts in the East and ends in the North-East direction. Figure 6 shows the $\mathrm{C} / \mathrm{M} 0+$ ratio averaged over each segment. On average the $\mathrm{C} / \mathrm{M} 0+$ ratio reaches it highest value in the southern segment (number 3 or direction S; see Fig. 6), that is in the same direction as the HI "bridge" that connects the LMC and the SMC. It thus seems that we have detected an increase of the $\mathrm{C} / \mathrm{M}$ ratio towards the bridge connecting the two Clouds and thus a lower metallicity. A lower metallicity has also been proposed by Muller et al. (2003) because of the detection of a ${ }^{12} \mathrm{CO}(1-0)$ emission region within the Magellanic Bridge $\left(\alpha=1^{\mathrm{h}} 56^{\mathrm{m}} 47^{\mathrm{s}}\right.$, $\left.\delta=-74^{\circ} 17^{\prime} 41^{\prime \prime}\right)$.

The same procedure has been applied to the latest type AGB stars selected according to the Loup et al. (2002) criteria; the result is shown in Fig. 7. In each ring the $\mathrm{C} / \mathrm{M}$ ratio has 


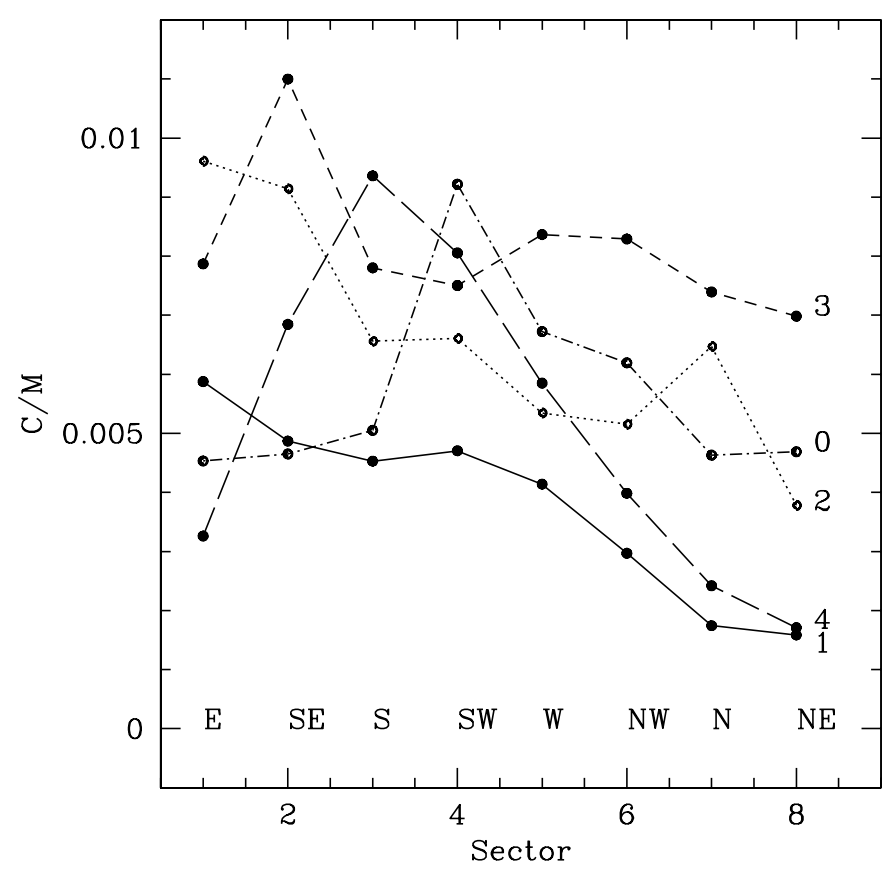

Fig. 6. The C/M ratio in the LMC, multiplied by the fractional area of the circular corona within which it has been calculated, is shown as a function of sector in each ring. Sector numbers correspond to increasing angle. The cardinal points indicate the starting direction of each sector. Each line represents the ratio in a ring: $\rho<2.5^{\circ}(0-$ dot-short-dashed line), $2.5^{\circ}<\rho<3.4^{\circ}(1-$ continuous line $), 3.4^{\circ}<$ $\rho<4.4^{\circ}(2-$ dotted line $), 4.4^{\circ}<\rho<5.5^{\circ}$ ( 3 - short-dashed line $)$ and $5.5^{\circ}<\rho<6.7^{\circ}$ (4-long-dashed line), respectively.

a peak. The innermost and the outermost ring have a peak in the SW region, while in the other rings the peak moves from $\mathrm{W}$ to $\mathrm{S}$ going from inside to outside the LMC. Note that the ratio $\mathrm{C} / \mathrm{M}$ is higher in Fig. 7 than in Fig. 6, because we excluded early-type $\mathrm{M}$-giants. The average $\mathrm{C} / \mathrm{M}$ ratio over the whole LMC $\left(0.19 \times A_{\mathrm{TOT}}\right)$ is about 2.68 which is very close to the value of $2.2 \pm 0.1$ obtained by Blanco \& McCarthy (1983) selecting carbon stars and giants of M6+ spectroscopically.

\subsection{Calibration of $\mathrm{C} / \mathrm{M}$ in terms of $[\mathrm{Fe} / \mathrm{H}]$}

In this section we quantify the $\mathrm{C} / \mathrm{M}$ ratio in terms of $[\mathrm{Fe} / \mathrm{H}]$ by using measurements available in the literature for galaxies in the Local Group with different metallicity.

We took as a reference the work by Groenewegen (2002). He lists the metallicity attributed to most of the galaxies in the Local Group with the number of carbon- and oxygenrich AGBs stars. We considered only those galaxies for which AGBs were detected ( $\mathrm{O}$ and $\mathrm{C}$ ). Figure 8 shows the decimal logarithm of the $\mathrm{C} / \mathrm{M}$ ratio versus metallicity. There is a clear correlation and the solid line indicates the best fit through the points.

If we use the same relation to quantify the spread in metallicity derived in the previous sections for the Magellanic Clouds, using the values corresponding to the lower and higher contours, we obtain about 0.75 dex between the central area and the outermost ring. This value is in agreement with the spread inferred from the metallicity of the star clusters in the

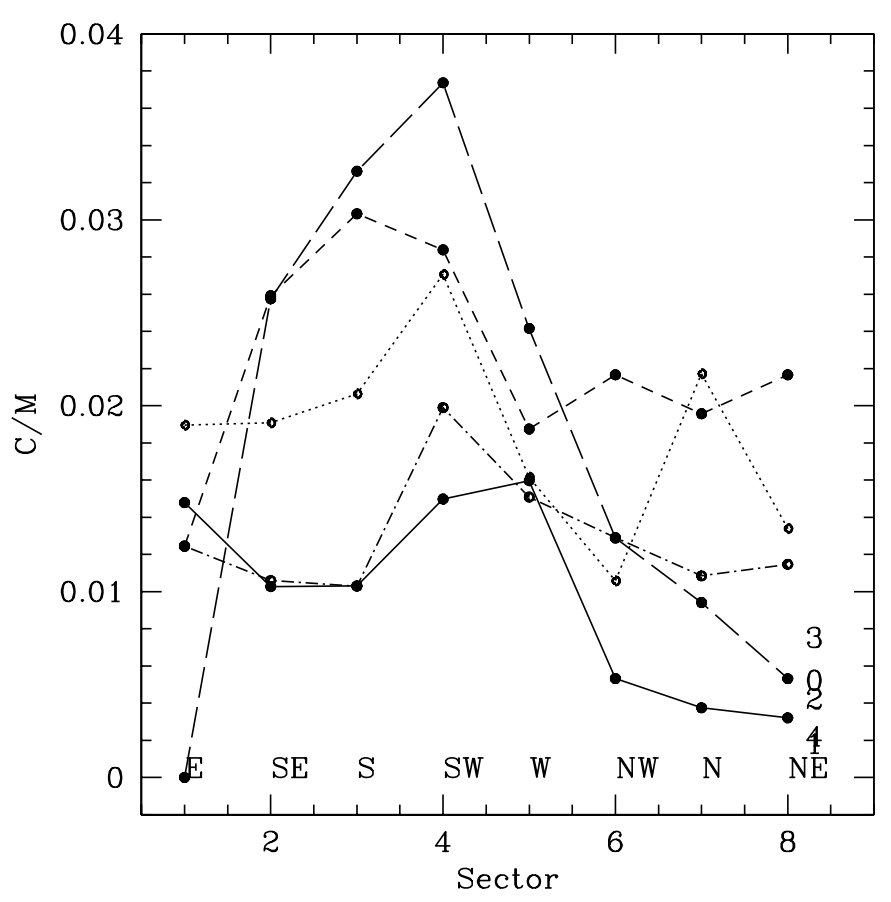

Fig. 7. Same as Fig. 5 but with a different selection of stars (see text).

LMC (Kontizas et al. 1993). The error on the linear fit is about 0.1 dex and we believe that the error on the $\mathrm{C} / \mathrm{M}$ ratio is negligible due to the large number of AGBs involved and to the completeness of detection. However, the ratio depends on the selection criteria used (cf. Table 2, Cols. 6-7). Mouhcine \& Lançon (2003) have shown that different star formation histories lead to non-linear curves in diagrams such as Fig. 8, but for populations older than $1 \mathrm{Gyr}$ the models merge into a common sequence. Here, we are not giving an absolute value for the metallicities in the Clouds because with the photometric data available at the moment we cannot determine the zero-point of the $\mathrm{C} / \mathrm{M}$ versus metallicity relation. The metallicity spread detected in the MCs agrees with the spread measured in Local Group dwarf galaxies (e.g. Shetrone et al. 2001, 2003; Tolstoy et al. 2003). However, the spread in metallicity may influence the fit in Fig. 8.

\subsection{Previous studies on abundance gradients in the MCs}

The indication of a metallicity gradient in the LMC and of a range of metallicities in the SMC obtained in this work is based on two fundaments: (i) our survey of the MCs is complete and (ii) our photometry allows us a statistical identification of the AGB stars of different atmospheric abundances. Previous studies of varying metallicity have been quite localised. For example, Nikolaev \& Weinberg (2000) do not find an indication of a radial metallicity gradient in the LMC using isochrone fitting to the red giant branch located in a centre $\left(\alpha=5^{\mathrm{h}} 24^{\mathrm{m}}\right.$, $\left.\delta=-69.8^{\circ}\right)$ and in an outer $\left(\alpha=6^{\mathrm{h}} 12^{\mathrm{m}}, \delta=-67.5^{\circ}\right)$ field of the LMC, of $2^{\circ} \times 1^{\circ}$ in size. Comparing the location of these fields with Fig. 3, our data confirm that between these fields there is no significant difference in abundances.

The most extensive work done in the eighties is that of Blanco \& McCarthy (1983). They surveyed the SMC and 


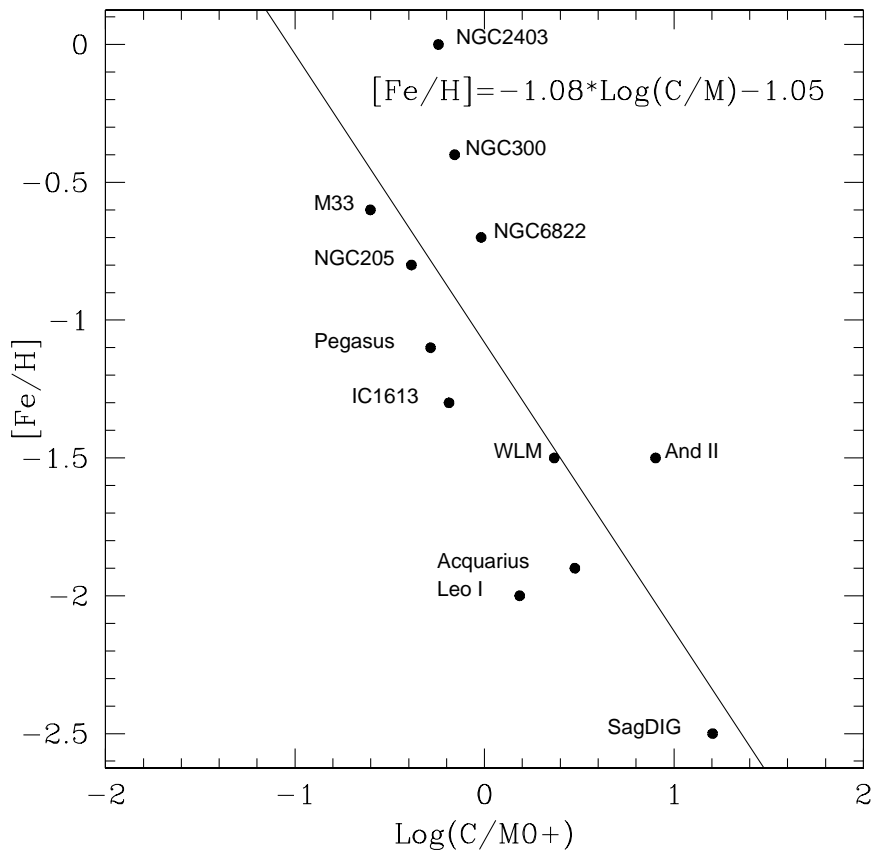

Fig. 8. Relation between the metallicity $([\mathrm{Fe} / \mathrm{H}])$ and the decimal logarithm of the $\mathrm{C} / \mathrm{M}$ ratio for $\mathrm{M} 0+$ stars. Data are taken from Groenewegen (2002). The solid line is a linear fit through the data.

the LMC down to a limiting magnitude of $I=17$ in 37 and 52 fields, respectively, of 0.12 square degrees each. Their survey is $95 \%$ complete for C-type and M5+-type AGBs while the numbers of M2-M4 stars may be in error by as much as $20 \%$. They found that the $\mathrm{C} / \mathrm{M}$ ratio in the LMC does not vary appreciably away from the central regions while in the SMC the ratio may decrease towards the peripheral regions. They further investigated the distribution of the ratios by binning the number of AGBs C- and O-rich in three groups: central, intermediate and outer. They concluded that there is no apparent gradient in the LMC while in the SMC a lower ratio in the outer field was confirmed. Note that these ratios include $\mathrm{O}-$-rich AGB stars of M6+ and that the variation of the $\mathrm{C} / \mathrm{M}$ ratio in the SMC disappears if also M2-M4 stars are included. The most important thing to notice is that their LMC fields are limited to $-65^{\circ}<\delta<-74^{\circ}$ and the highest value of the $\mathrm{C} / \mathrm{M}$ ratio that we detect is at about $\delta=-75^{\circ}$ (Fig. 3). Reid \& Mould (1985) took spectra of all the stars in six fields in the northern part of the LMC, three of which contain a Blanco et al. (1980) field. Reid \& Mould concluded that if a radial variation of the $\mathrm{C} / \mathrm{M}$ ratio exists, then the Bar is less enriched in heavy elements than the outer regions. Comparing the location of their analysed fields with Fig. 3, we find that these fields are not representative of the radial distribution.

A positive indication of a metallicity spread in the Magellanic Clouds comes from Luck \& Lambert (1982) who concluded, from measurements in Cepheids and non-variable supergiants that the $[\mathrm{Fe} / \mathrm{H}]$ ratio in the $\mathrm{SMC}$ has an abundance dispersion consistent with the errors while in the LMC a real dispersion is possible. In addition Spite et al. (1989a,b) suggested that inhomogeneities in the abundances derived from $\mathrm{F}$ and $\mathrm{G}$ super giants are due to a low metallicity in the halo and a progressively increasing metallicity in the disk.
Kontizas et al. (1993) studied a sample of 94 star clusters in the LMC of intermediate age (between 0.5 and 3 Gyr). They found that the outermost clusters are significantly metal poorer than those at the centre. Their outer system coincides with our outermost region (see their Fig. 1) where we detect the highest $\mathrm{C} / \mathrm{M}$ ratios.

\subsection{Metallicity gradients in other galaxies of the Local Group}

There is evidence, though somewhat controversial, that the metallicity is a decreasing function of radius in other galaxies in the Local Group.

In our Galaxy the metallicity gradient is present among clusters (Friel 1995). Note that clusters do not usually contain $\mathrm{C}$ stars even if they are metal poor. This is strictly an age effect and the $\mathrm{C} / \mathrm{M}$ is directly correlated with the intermediate age stars. Andrievsky et al. (2002) recently concluded that a linear fit to the $[\mathrm{Fe} / \mathrm{H}]$ data of Galactic Cepheids from 4 to $12 \mathrm{kpc}$ produces a grandient of $-0.06 \mathrm{dex} \mathrm{kpc}^{-1}$.

Fields in the halo and in the centre of M31 were observed in $B$ and $V$, the colour variation corresponds to about 0.2 dex in $[\mathrm{Fe} / \mathrm{H}]$ (van den Bergh \& Pritchet 1992). In M31 Brewer et al. (1995) found an increase of the $\mathrm{C} / \mathrm{M}$ ratio with galactocentric distance by studying seven fields spaced along the M31's SW semi-major axis. On the other hand, Freedman \& Madore (1990) argue that because the period-luminosity relation of Cepheids does not vary with radius in M31 there is no abundance gradient. Globular clusters in M31 show a spread in metallicity within $5 \mathrm{kpc}$ from the centre but metal rich clusters exist in the outer halo (Huchra et al. 1991).

Nitrogen and oxygen elements present a strong radial gradient in M33 (Smith et al. 1993). In IC 1613 a slight C/M gradient from the center outward has been detected. Stars of the two spectral types were selected using the $(\mathrm{CN}-\mathrm{TiO}) /(R-I)$ diagram. M0+ stars were also included (Albert et al. 2000).

In the dwarf spheroidals NGC 147, NGC 187 and NGC 205 the width of the RGB is the major indication of a metallicity spread which is often larger in the centre of the galaxies than in the outer parts (Han et al. 1998; Martínez-Delgado \& Aparicio 1998; Mould et al. 1984). The Sculptor galaxy presents a strong radial population effect (Hurley-Keller et al. 1999) and it would be interesting to know if and how this is associated to a gradient in metallicity.

\section{Conclusions}

We have derived $\mathrm{C} / \mathrm{M}$ ratios in the Magellanic Clouds from AGB stars selected in colour-magnitude diagrams obtained from the DCMC catalogue. This value is a simple, but robust indication of the metallicity of the AGB population. It varies considerably across the surface of both galaxies. In the LMC we find a increase of the ratio $\mathrm{C} / \mathrm{M} 0+$ in at the inside of the elliptical distribution of the AGB star. The distribution of the $\mathrm{C} / \mathrm{M}$ values, however, are rather clumpy and that has prevented previous authors from detecting such a gradient. A more detailed analysis of the $\mathrm{LMC} \mathrm{C/M}$ ratio shows that towards the 
direction of the bridge connecting the two Clouds the ratio $\mathrm{C} / \mathrm{M} 0+$ is still higher.

The range of variation of the $\mathrm{C} / \mathrm{M}$ ratio has been calibrated in terms of $[\mathrm{Fe} / \mathrm{H}]$; in the $\mathrm{LMC}$ it corresponds to a spread in metallicity of $0.75 \mathrm{dex}$. The same spread has been found in the SMC and it approximately corresponds to the range of values derived in intermediate age LMC star clusters. The SMC has on average a lower metallicity than the LMC and the distribution of regions of high $\mathrm{C} / \mathrm{M} 0+$ shows no systematic behaviour.

To conclude, it is important to remember that the variation of metallicity found is valid for the epoch when these giants formed and it is not necessarily valid today or for other epochs better traced by other stellar populations or gas. On the other hand in view of the recent results by Mouhcine \& Lançon (2003) the C/M ratio measures both the metallicity of the carbon star progenitors and the present interstellar medium metallicity, unless the local composition has been strongly altered by the presence of a Bar or other interactions.

Acknowledgements. The authors thank Martino Romaniello, Luca Pasquini and Vanessa Hill for useful discussions during the preparation of this article.

\section{References}

Albert, L., Demers, S., \& Kunkel, W. E. 2000, ApJ, 119, 2780

Andrievsky, S. M., Kovtyukh, V. V., Luck, R. E., et al. 2002, A\&A, 396, 641

Blanco, V. M., \& McCarthy, M. F. 1983, AJ, 88, 1442

Blanco, V. M., McCarthy, M. F., \& Blanco, B. M. 1980, ApJ, 242, 938

Brewer, J. P., Richer, H. B., \& Crabtree, D. R. 1995, AJ, 109, 2480

Cioni, M.-R. L., Loup, C., Habing, H. J., et al. 2000a, A\&AS, 144, 235

Cioni, M.-R. L., Habing, H. J., \& Israel, F. P. 2000b, A\&A, 358, L9

Cioni, M.-R. L., van der Marel, R. P., Loup, C., \& Habing, H. J. 2000c, A\&A, 359, 601

Cioni, M-R. L. 2001a, AGB stars and other red gaints in the Magellanic Clouds, Ph.D. Thesis, University of Leiden

Cioni, M.-R. L., Marquette, J.-B., \& Loup, C. 2001b, A\&A, 377, 945

Cioni, M.-R. L. 2002, Workshop on: Mass-loosing Pulsating Stars and their Circumstellar Matter, Sendai (Japan) [astro-ph/0207619]
Cook, K. H., Aaronson, M., \& Norris, J. 1986, ApJ, 305, 634

Epchtein, N., De Batz, B., Capoani, L., et al. 1997, The Messenger, 87,27

Feast, M., \& Whitelock, P. 1994, MNRAS, 269, 737

Fluks, M. A., Plez, B., The, P. S., et al. 1994, A\&AS, 105, 311

Freedman, W. L., \& Madore, B. F. 1990, AJ, 365, 186

Friel, E. D. 1995, ARA\&A, 33, 381

Frogel, J. A., \& Blanco, V. M. 1990, ApJ, 365, 168

Glass, I. S., Ganesh, S., Alard, C., et al. 1999, MNRAS, 308, 127

Groenewegen, M. A. T. 2002, Ringberg Castle Workshop [astro-ph/0208449]

Han, J. L., Beck, L., \& Berkhuijsen, E. M. 1998, A\&A, 335, 1117

Huchra, J. P., Brodie, J. P., \& Kent, S. M. 1991, AJ, 370, 495

Hurley-Keller, D., Mateo, M., \& Grebel, E. K. 1999, AJ, 523, L25

Kontizas, M., Kontizas, E., \& Michalitsianos, A. G. 1993, A\&A, 269, 107

Kontizas, E., Dapergolas, A., Morgan, D. H., \& Kontizas, M. 2001, A\&A, 369, 932

Iben, I., Renzini, A. 1983, ARA\&A, 21, 271

Jorissen, A. 2003, in Asymptotic Giant Branch stars, ed. H. J. Habing, \& H. Olofsson (Springer Verlag), in press

Loup, C. 1999, IAU Symp., 190, 328

Loup, C., Cioni, M.-R. L., Duc, P. A., et al. 2002, A\&A, submitted

Luck, R. E., \& Lambert, D. L. 1982, ApJ, 256, 189

Martínez-Delgado, D., \& Aparicio, A. 1998, AJ, 115, 1462

Mikami, T. 1978, Tokyo Astronomical Observatory, Annals, Second Ser., $17,1,1$

Mouhcine, M., \& Lançon, A. 2003, MNRAS, 338, 572

Mould, J., Kristian, J., \& Da Costa, G. S. 1984, AJ, 278, 575

Muller, E., Staveley-Smith, L., \& Zealey, W. J. 2003, MNRAS, 338, 609

Nikolaev, S., \& Weinberg, M. D. 2000, AJ, 542, 804

Rebeirot, E., Martin, N., Mianes, P., et al. 1983, A\&AS, 51, 277

Reid, N., \& Mould, J. 1985, ApJ, 299, 236

Shetrone, M. D., Côté, P., \& Sargent, W. L. W. 2001, ApJ, 548, 592

Shetrone, M., Venn, K. A., Tolstoy, E., et al. 2003, AJ, 125, 684

Smith, R. C., Kirschner, R. P., Blair, W. P., et al. 1993, AJ, 407, 564

Spite, F., Spite, M., \& Francois, P. 1989a, A\&A, 210, 25

Spite, M., Spite, F., \& Barbuy, B. 1989b, A\&A, 222, 35

Tolstoy, E., Venn, K. A., Shetrone, M., et al. 2003, AJ, 125, 707

van den Bergh, S., \& Pritchet, C. J. 1992, IAU Symp., 149, 161

van der Marel, R. P., \& Cioni, M.-R. L. 2001, AJ, 122, 1807

Westerlund, B. E. 1999, The Magellanic Clouds, Cambridge Astrophys. Ser. (Cambridge University Press) 